\title{
Design of Optical Fiber Fabry-Perot Sensors Based on Intensity and Phase with Parallel Processing
}

\author{
Hui Peng*
}

Hunan University of Finance and Economics, Hunan ChangSha, 410205, China

\begin{abstract}
For fiber Fabry-Perot sensor vulnerable to external parameters such as electromagnetic, noise and displacement impact, reducing the problem resilience, research and design new fiber Fabry-Perot sensor with parallel processing capabilities based on the intensity and phase of perception. First, according to various types of external parameters and the degree of interference, established the intensity and phase-oriented demodulation algorithm then gives a fiber Fabry-Perot microcavity sensor performance analysis model is then proposed for the fiber Fabry-Perot sensor has a solution parallel processing algorithms tune transformation, and finally developed a fiber Fabry-Perot sensor has strong adaptability and measurement effects. Experimental results show that the single phase demodulation and intensity demodulation fiber Fabry-Perot sensor design compared to the proposed sensor design has obvious advantages in accuracy and performance of the strain.
\end{abstract}

Keywords: Intensity, optical fiber fabry-perot sensor, parallel processing, phase.

\section{INTRODUCTION}

The internal optical fiber Fabry Perot sensor design has two story high reflection films. The parallel arrangement formed a narrow cavity, which is shown in Fig. (1). L denotes the micro cavity length. When the external beam through the optical refraction to the micro cavity, the emission film interlayer reflection would be returned. At the same time, there is the beam multiplexing interference and distortion phenomenon, etc. The detection value could be obtained by checking the output signal according to the micro cavity length. The optical fiber Fabry Perot cavity would be deployed in the monitoring object after connecting the fiber. The status of output beam could be changed and the parameter settings status would be perceived on the basis of sensing the small changes of the environment with Fabry Perot strong induction, which has been used in the fields of bridge strain [1] and anti-shock grating strain sensor [2]. There are some research results of the demodulation, strain, anticorrosion and so on.

The non-scanning the cavity length information is used to decode the non-scanning Multi-channel fiber optic Fabry Perot [3], the authors researched the optical model of demodulation system of optical fiber Fabry Perot sensor. For designing the transverse, longitudinal and tangential regulation of strain measurement adaptive direction, the article [4] proposed the Fiber Bragg grating strain sensor based on the similar material model. L J Jiang et al. [5] researched the cavity length analysis method by approximating the Fiber optic Fabry Perot pressure sensing to the signal envelope spectrum curve signal spectrum curve. Y J Rao et al. [6] proposed the optical fiber Fabry Perot sensor with the germanium chemical corrosion of boron has the advantages of simple structure, portable and low cost of Co doped fiber fabrication.

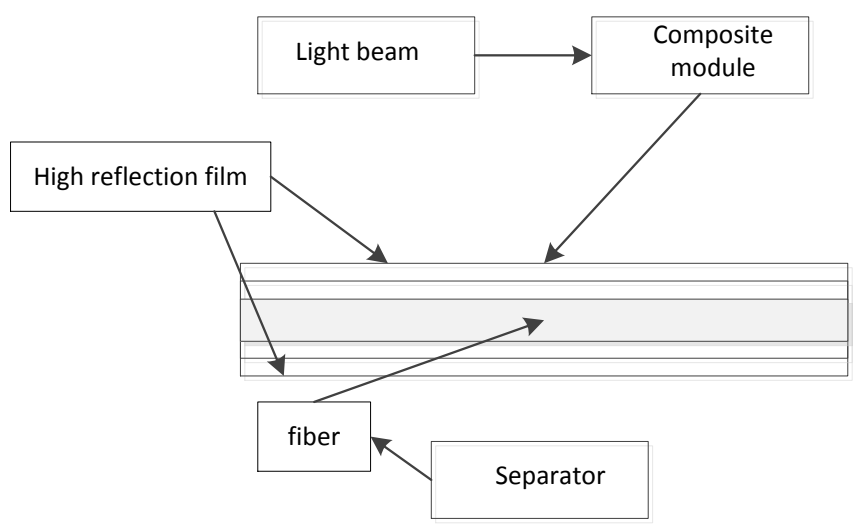

Fig. (1). Optical fiber fabry perot sensor structure.

Huizu Lin et al. [7] analyzed the phase carrier generated by the mode crosstalk and the embedded time division optical fiber Fabry Perot sensor system by multiple reflection. $\mathrm{L} \mathrm{H} \mathrm{Li} \mathrm{et} \mathrm{al.} \mathrm{[8]} \mathrm{proposed} \mathrm{the} \mathrm{small} \mathrm{volume} \mathrm{and}$ low cost optical system based on the non-scanning correlation demodulation cavity length information. Yong Zhao et al. $[9,10]$ proposed the FP optical fiber magnetic field sensor with temperature compensation model by combining the characteristics of Fiber Prague grating temperature sensing.

In order to reducing the influence with the Electromagnetic noise and the external parameters, such as displacement on the Fiber Fabry Perot sensor strain capacity, there are the following contributions in our research. First, the Analysis of performance model and the combined demodulation scheme were proposed based on the influence characteristics of intensity and phase demodulation of the long Fabry Perot cavity. Second, we designed the combined demodulation conversion circuit based on the intensity and 
phase orientation. Finally, the new optical fiber Fabry Perot sensor was researched on the view of the above results.

\section{MICRO CAVITY PERFORMANCE ANALYSIS MODEL}

The communication performance of optical fiber Fabry Perot sensor is mainly affected by the length of cavity, which cause that the output signal beam of demodulation has different selectivity. However, the status of cavity length is easily to be disturbed by the external parameters, which include the external force, optical fiber Fabry Perot displacement, deformation, environment temperature, voltage and current, etc. The phase $P_{H}$ and intensity $I_{N}$ of optical fiber Fabry Perot sensor with the process of modulation and demodulation have the most obvious interference from the above parameters. The Fabry Perot interferometer is shown in Fig. (2).

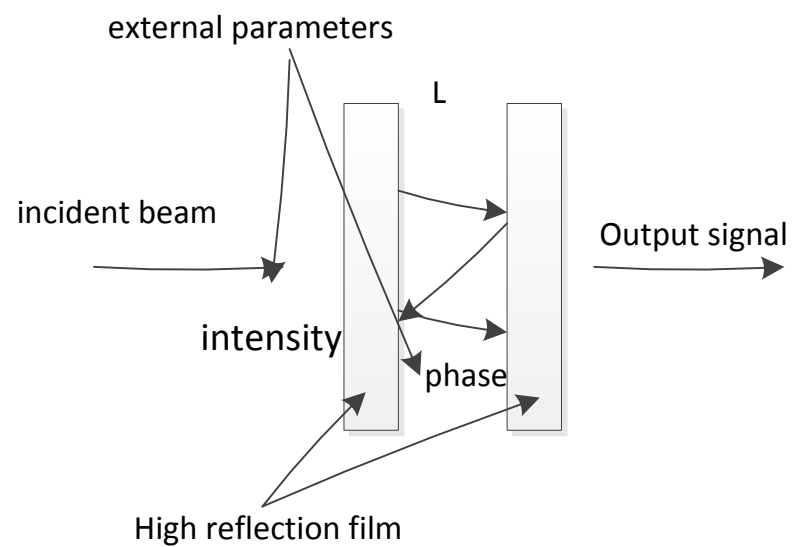

Fig. (2). Process of external parameters interference.

The output signal $I_{R}$ could be obtained by the emission and transmission combined with the external parameters after the phase demodulation, which is given by formula (1).

$$
I_{R}=\frac{2 d \cos \alpha}{1+\cos \frac{1}{2} \alpha \sqrt{d^{2}-d_{0}^{2}}} P_{H}
$$

Here, let $\alpha$ denote the refraction radian which could be obtained by formula (2).

$$
\left\{\begin{array}{c}
\alpha=\frac{(1-d)^{\frac{3}{2}}}{1+d_{0}^{2}-\frac{3}{2} \cos \varphi} \\
\varphi=\frac{\pi}{4} \lambda \sqrt{\left|d^{2}-d_{0}^{2}\right|}
\end{array}\right.
$$

Here, let $\lambda$ denote the refraction beam wavelength and denote $\varphi$ the optical phase.

The optical signal received by the photoelectric detector is illustrated by formula (3) after the intensity demodulation.

$$
\left\{\begin{array}{c}
I_{R}=\frac{d(1-\cos (\varphi))}{\frac{I_{N}-\mu}{d_{0}}} \\
\mu=\alpha I_{N}
\end{array}\right.
$$

When the sender of the optical fiber Fabry Perot sensor select the modulation based on the phase or intensity parameters, the receiver could combine the phase and intensity to demodulate the optical signal, which is shown as formula (4).

$$
I_{R}=\frac{d^{2} d_{0} \cos \alpha(1-\cos (\varphi)) P_{H}}{I_{N}-\mu+\cos \frac{1}{2} \alpha \sqrt{d^{2}-d_{0}{ }^{2}}}
$$

After the modulation of combination of phase and intensity, the interference signal strength of external parameters would be growing after the optical fiber refractive by Fabry Perot strain. Hence, the selfcompensation algorithm of fiber optic Fabry Perot refractive radian and Transmittance perception would be applied in our design scheme, which is demonstrated by Figs. $(3,4)$ gives the equivalent circuit of micro controller.

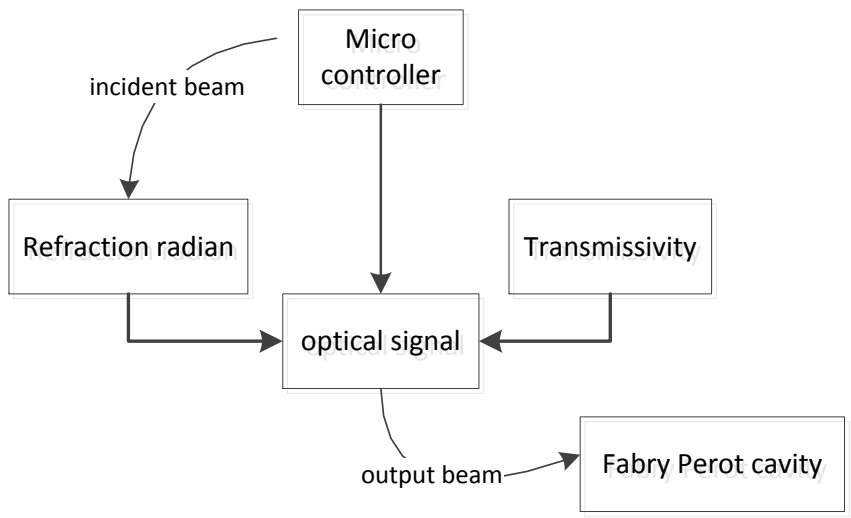

Fig. (3). Self-compensation algorithm of fiber optic fabry perot.

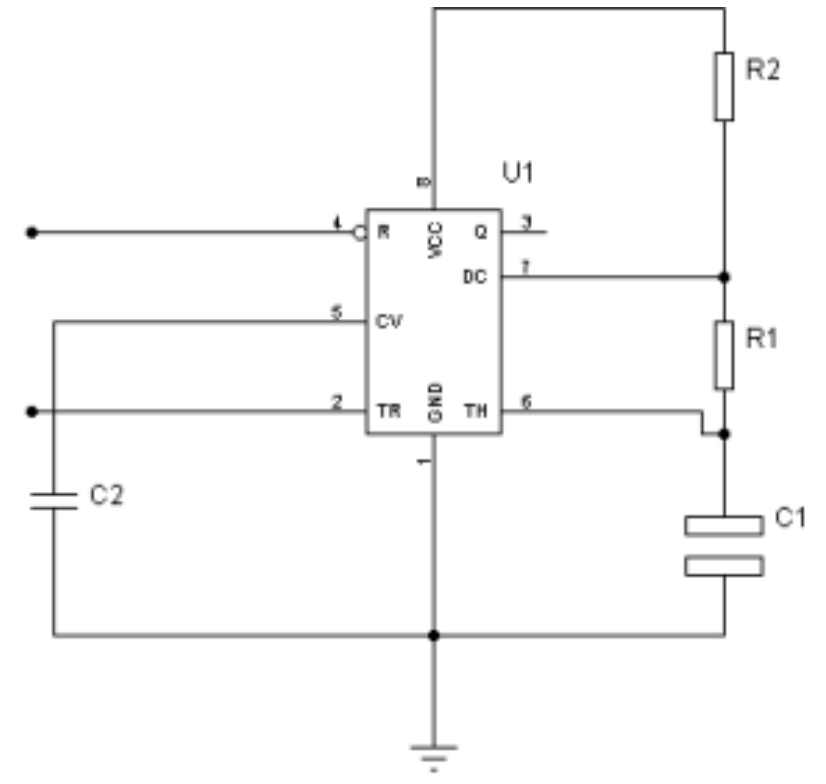

Fig. (4). Equivalent circuit of micro controller. 
In Fig. (4), the wave rectifier circuit is composed by resistor R1 and capacitance $\mathrm{C} 1$, which is used to eliminate external interference in electromagnetic field parameters. R2 is the microcontroller internal resistance. The function of working circuit of DC voltage stabilizing is implemented by the charge and discharge of the capacitor $\mathrm{C} 2$.

Based on the analysis results of formula (1), (2), (3) and (4), the change regulation of optical signal intensity of micro cavity fiber Fabry Perot sensor based on the phase and intensity belonged with wavelength and cavity length is illustrated by Fig. (5).

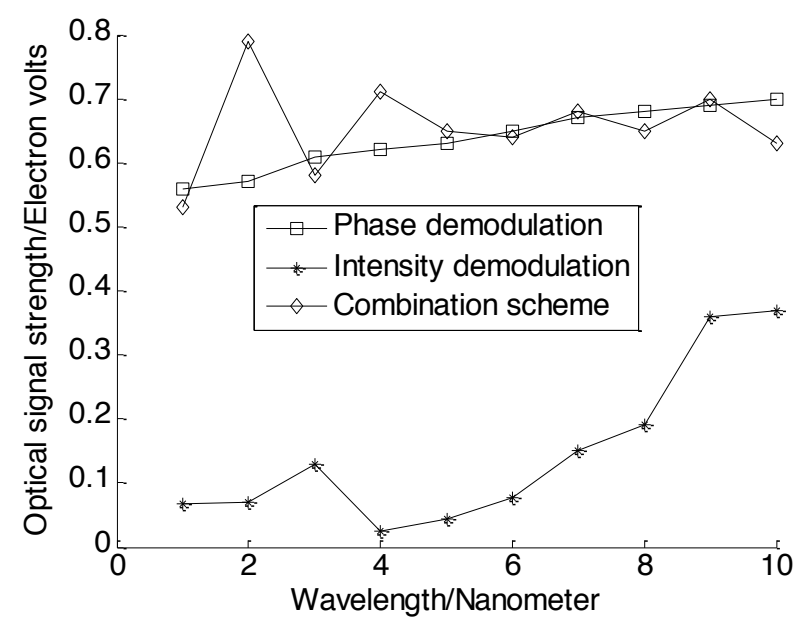

(a) wavelength

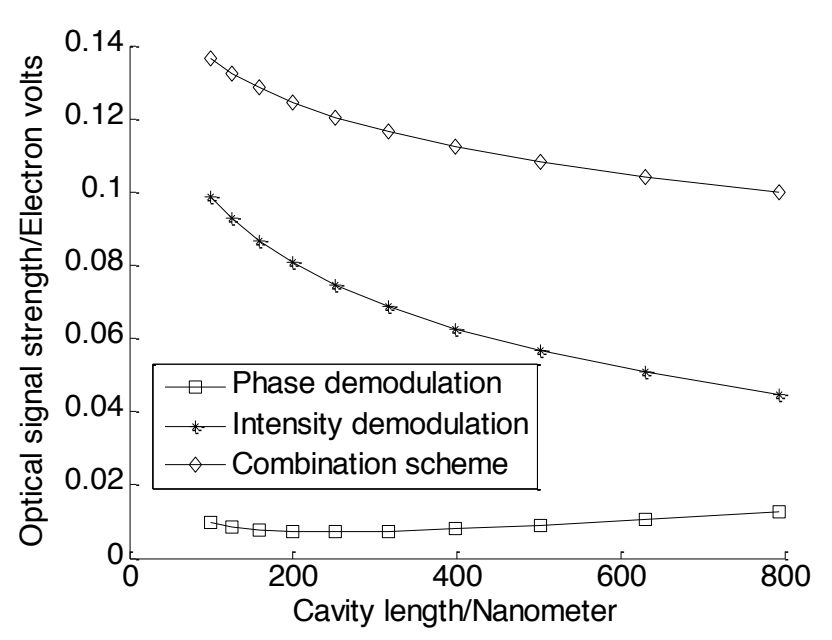

(b) Cavity length

Fig. (5). Analysis of the optical signal strength properties.

From Fig. (5a), we found that Optical signal strength of three demodulation scheme gradually increased with the increasing of wavelength. However, some points of the combination scheme is lower than one of intensity demodulation scheme, because of the missing of some signal affected by the external parameters. In general, the performance of the combination scheme is superior to the other schemes. The superiority and rationality of the combination scheme has been verified from the angle of cavity length by Fig. (5b).

\section{OPTICAL FIBER FABRY PEROT SENSOR BASED ON INTENSITY AND PHASE}

On the basis of the above section of analysis model of fiber optic Fabry Perot micro cavity properties, we proposed the structure of Optical fiber Fabry Perot sensor aware the phase, intensity and outside interference parameters, which is given by Fig. (6).

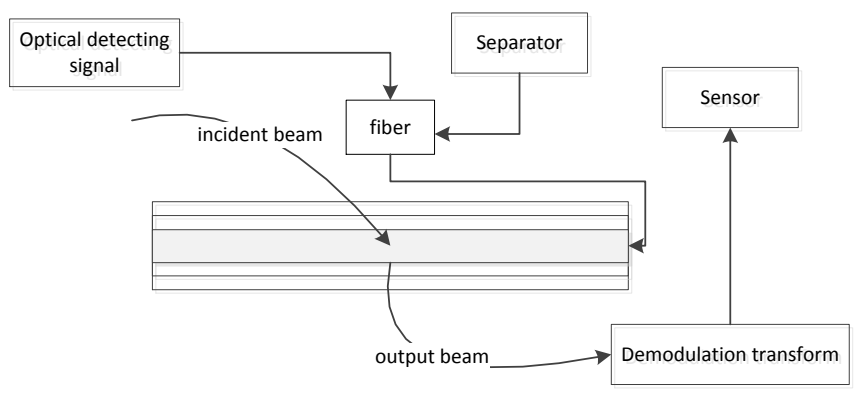

Fig. (6). Structure of optical fiber fabry-perot sensor.

The output beam would be sent to the Optical fiber Fabry Perot sensor after the demodulation transforms. This operation is conducive to adjust the phase demodulation and intensity demodulation scheme of the proposed combination scheme, as well as select the current best demodulation scheme for improving the accuracy and efficiency. The demodulation conversion circuit is shown in Fig. (7).

Optical signal strength $I_{R}$ after transform demodulation is given by formula (5).

$$
I_{R}=A+B \cos \left(\frac{\pi}{\mu} \alpha\right)
$$

Here, $\mathrm{A}$ is the demodulation gain based on the phase demodulation. $\mathrm{B}$ is the demodulation gain of Fiber optic Fabry Perot according to intensity demodulation. Parallel processing demodulation scheme based on the combination of intensity and phase is proposed to improve the demodulation efficiency and relieve stagnation effect brought by the transform demodulation. The optical signal intensity can be calculated by the formula (6).

$$
\left\{\begin{array}{c}
I_{0}(\lambda)=I_{d_{0}} \sqrt{\frac{\lambda-L}{P_{H}} \mid} \\
I_{1}(L)=I_{d_{0}} e^{\frac{\sqrt{\left(d^{2}-d_{0}^{2}\right)}}{-\mu-\gamma \mid}} \\
I_{R}=\left|I_{0}(\lambda) I_{1}(L)-2 \frac{P_{H}}{I_{N}}\right|_{-\lambda}^{L} 2 d I_{N}\left[1-\cos \left(\frac{1}{2} \pi \alpha\right)\right]
\end{array}\right.
$$

In the practical application, there are the following issues with the parallel process of optical fiber Fabry Perot sensor, which should be concerned about.

(1) Parallel processing time frequency intervals should satisfy the following relations.

$$
f(\delta)=\frac{\alpha \gamma}{\gamma(\alpha+\lambda)}
$$




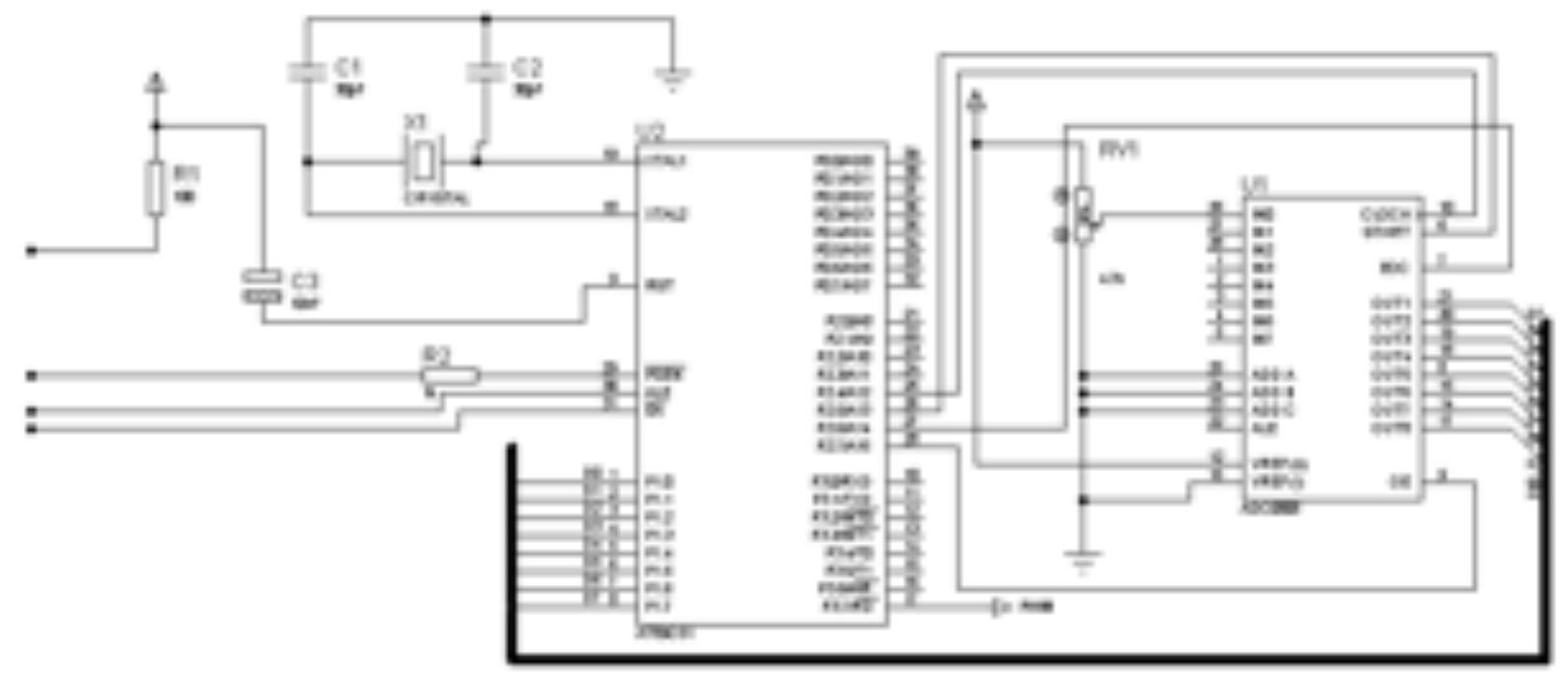

Fig. (7). Demodulation conversion circuit.

(2) The high frequency noise interference should be smoothed in the Parallel processing progress, which could be applied with formula (8).

$$
I(k)=\sum_{n=1}^{M} I_{R}(n) \ln \left(-j \frac{1}{2} \pi k\right)
$$

Here, the optical signal after each round of high frequency noise interference could be obtained by formula (4).

(3) Detuning case for optical signal demodulation could be defused according to formula (9).

$$
I_{R}(t)=\int_{-\lambda}^{\lambda} F(\sigma, t) f(\delta, t) d t
$$

\section{PERFORMANCE EVALUATION}

In this work, the proposed opportunistic cooperative QoS control protocol based on GOP-length and video Framedensity (OCQ-GF) and cooperative QoS control protocol (CQ) are simulated, analyzed and evaluated with two group experiments, which include four metrics: packet error rate, average delay, Peak Signal to Noise Ratio (PSNR) and throughput.

There are one cube closed environment in our experiments, size of which is $500 \mathrm{~mm} * 600 \mathrm{~mm}$ and $* 550$ $\mathrm{mm}$. The light beam wavelength belongs to the interval $[1500,1600]$ nanometer, step of which is 50 nanometer. We design 2 groups of experiments. The temperature inside the cube is measured in first experiment with the optical fiber Fabry Perot sensor. In the second experiment, we study the strain capacity of optical fiber Fabry Perot sensor in static and dynamic conditions.

Fig. (8) gives the experimental process. Here, we consider the light layout, Fabry Perot microcavity length selection, intensity and phase demodulation sensing module, as well as transformation module. Other parameters is shown in Table 1. In our experiments, let PH-Sensor denote the
Optical fiber Fabry Perot sensor based on phase demodulation. Let IN-Sensor denote the Optical fiber Fabry Perot sensor based on intensity demodulation. Let PIPSensor denote the proposed parallel processing optical fiber Fabry Perot sensor based on demodulation transformation and combination of Intensity and phase. The experimental results are shown in Fig. (9).

Table 1. Settings of experiment parameters.

\begin{tabular}{|c|c|}
\hline Parameters & Value \\
\hline \hline spectral width & $1525 \mathrm{~nm}$ \\
\hline coherence length & $1515 \mathrm{um}$ \\
\hline Spectral coefficients & 2.8 \\
\hline relative strength of the light signal & 0.52 Micro watts per nm \\
\hline
\end{tabular}

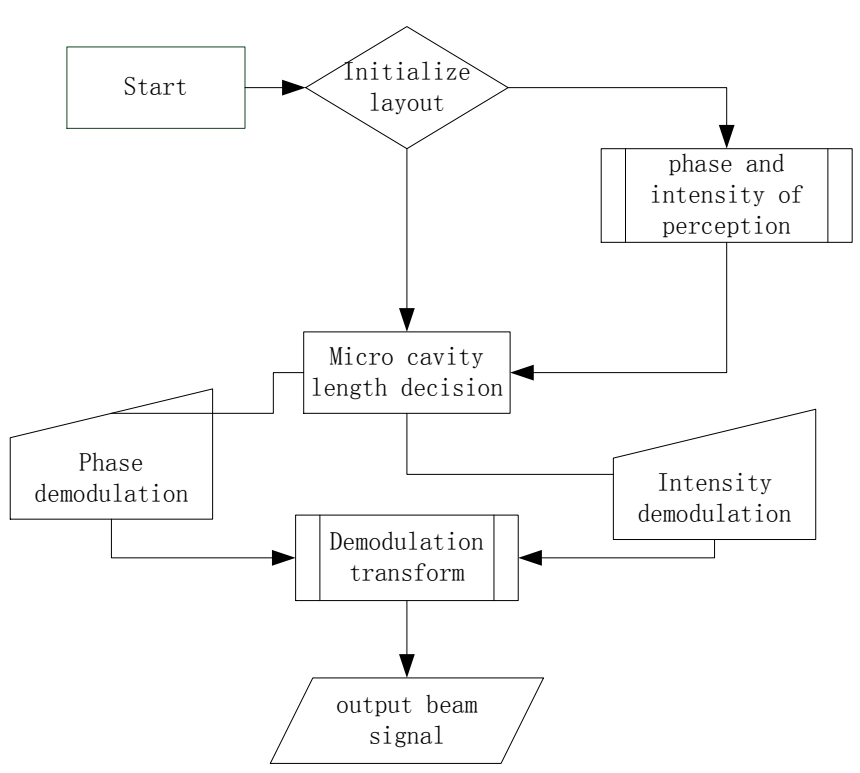

Fig. (8). Experimental process. 


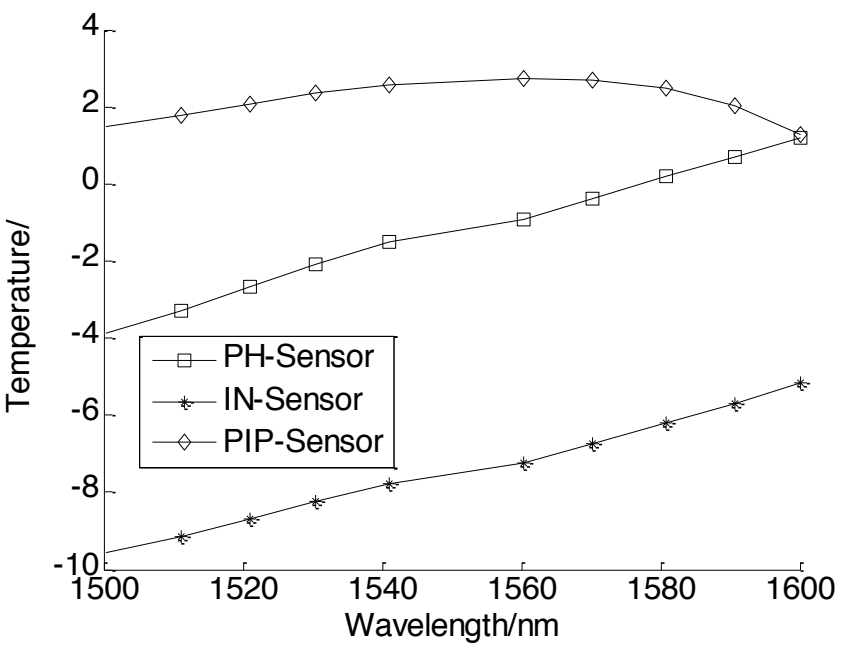

(a) wavelength

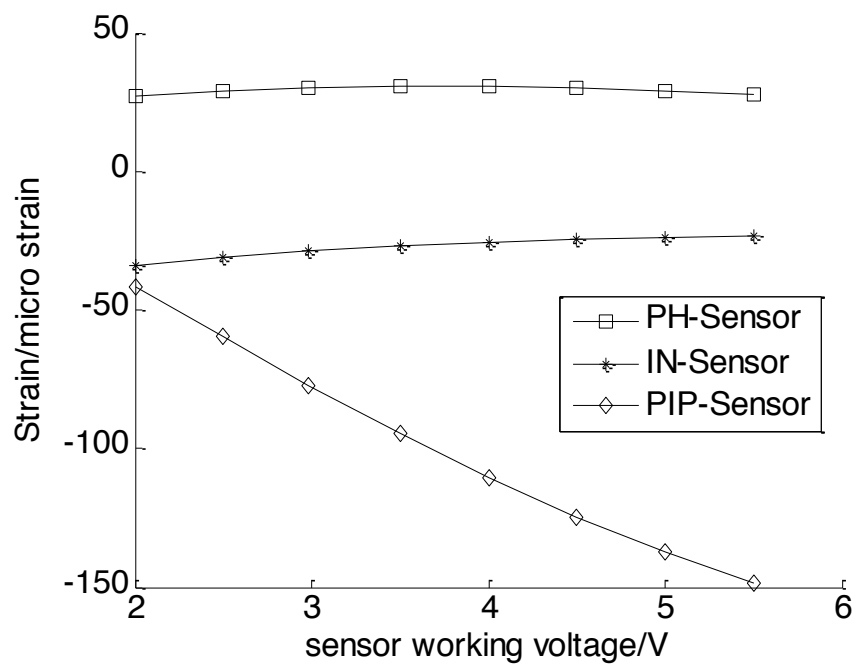

(b) Sensor working voltage

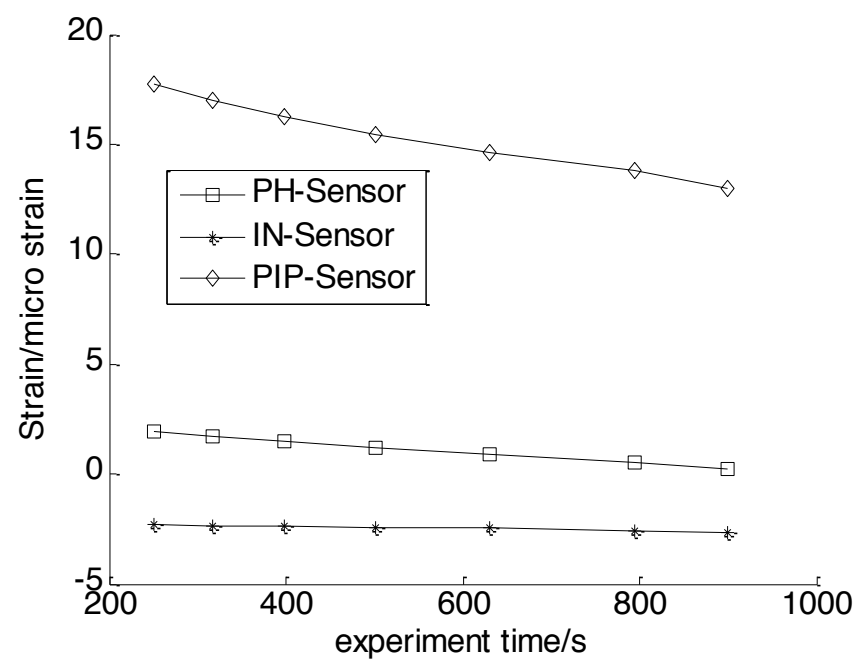

(c) Time

Fig. (9). Performance evaluation.

The temperature value in the cube along with the change of wavelength is shown in Fig. (9a). We found that the value of the proposed scheme is close to the real value. Other, the performance of the proposed scheme is similar to the PHSensor scheme when wavelength is large enough.

Fig. (9b) gives the statistical performance of optical fiber Fabry Perot sensor static strain in the second set of experiments. The PH-Sensor scheme and IN-Sensor scheme are not sensitive to the sensor working voltage. However, the proposed scheme of strain value and the actual value strain is the most consistent. The performance results of optical fiber Fabry Perot sensor with dynamic conditions is given by Fig. (9c). Here, there are great difference between the strain detection value of PH-Sensor scheme and IN-Sensor scheme and actual value. That because that the design of Optical fiber Fabry Perot sensor is lack of real-time demodulation switching capacity and parallel processing ability, which bring about the poor performance of strain. However, the proposed scheme has better ability of self-compensation. Performance of the proposed sceme is better than the above two kinds of schemes.

\section{CONCLUSION}

It is well known that the Optical fiber Fabry Perot sensor is vulnerable to various types of external parameters interference degree. We proposed the optical fiber Fabry Perot sensor including the ability of parallel processing based on the sensor strain condition. First, we designed the analytical model of Fabry Perot cavity with the external parameters, intensity and phase. Second, the combined demodulation scheme and transform circuit is proposed to make up the disadvantages of composite beam and combined demodulation. Finally, we researched and developed a new type of optical fiber Fabry Perot sensor. The experimental results verify the proposed scheme design is superior to single phase demodulation and intensity demodulation of the optical fiber Fabry Perot sensor, such as temperature measurement, static performance and dynamic performance of strain.

\section{CONFLICT OF INTEREST}

The authors confirm that this article content has no conflict of interest.

\section{ACKNOWLEDGEMENTS}

This work is supported by the construct program of the key discipline in Hunan province.

\section{REFERENCES}

[1] H. Lin, L. Ma, Z. Hu, Q. Yao, and Y. Hu, "Multiple reflections induced crosstalk in inline TDM fiber Fabry-Perot sensor system utilizing phase generated carrier scheme," Journal of Lightwave Technology, vol. 31, pp. 2951-2958, 2013.

[2] L.-J. Jiang, J.-F. Jiang, T.-G. Liu, K. Liu, Y. Liu, and X. Liang, "Demodulation of cascade optical fiber fabry-perot pressure sensor," Acta Photonica Sinica, vol. 41, pp. 283-287, 2012.

[3] L. Jin-lan, W. Ning, and L. Jun-Shou, The optical characterization and experiments of fiber Fabry-Perot non-scanning coherent demodulation system based on SLED, In: Lasernal, pp. 43-45, 2014.

[4] L.-h. Li, Y. Zhu, N. Wang, T.-C. Gong, and Y. Li, "Analysis and experimental study on multi-channel optical fiber fabry-perot demodulation system," Acta Photonica Sinica, vol. 9, p. 006, 2013. 
[5] L. Li-hui, Z. Yong, and W. Ning, "Analysis of the optical fiber Fabry-Perot demodulation system with non-scanning correlation", In: Lasernal, pp. 26-28, 2014.

[6] Y.-J. Rao, Y. Guo, and Y. Gong, "Self-enclosed fiber-optic FabryPerot sensor based on etched B-Ge co-doped optical fiber," Journal of University of Electronic Science and Technology of China, vol. 41, pp. 227-231, 2012.

[7] J. Wang, S.-C. Li, Q.-M. Sui, J.-Y. Zhang, X.-G. Chen, and Z.-F. Wang, "Study of FBG strain sensors based on similar materiel in zonal disintegration model test," Journal of China Coal Society, vol. 37, pp. 1570-1575, 2012.
[8] X.-C. Xiong, Y. Zhu, Y.-M. Z. P. Fu, W.-M. Chen, and Y.-X. Zhang, "Fiber fabry-perot sensor and its application for monitoring bridge strain," Journal-Chongqing Jianzhu University, vol. 29, p. 48, 2007.

[9] J.-X. Yang, S.-J. Yu, and X. Chen, "Shock test for a fiber Bragg grating strain sensor on a SHPB," Journal of Vibration and Shock, vol. 1, p. 15, 2012.

[10] Y. Zhao, R.-Q. Lv, D. Wang, and Q. Wang, "Fiber optic fabryperot magnetic field sensor with temperature compensation using a fiber Bragg grating," In: IEEE Transactions on Instrumentation and Measurement, vol. 63, pp. 2210-2214, 2014.

Received: September 16, 2014

Revised: December 23, 2014

Accepted: December 31, 2014

(C) Hui Peng; Licensee Bentham Open.

This is an open access article licensed under the terms of the (https://creativecommons.org/licenses/by/4.0/legalcode), which permits unrestricted, non-commercial use, distribution and reproduction in any medium, provided the work is properly cited. 